with the movements of the globe by reason of the advancement of the fornix.

There is no danger of ptosis following the operation as above described. On the contrary the trachomatous ptosis so often present is relieved, the lid is raised and the palpebral aperture widened. There is a tendency unless the central stitch be put in rather higher than the others for the centre of the lid margin to be drawn up in a shallow concave notch. This is undesirable from a cosmetic point of view.

The operation wound heals rapidly and the raw area quickly becomes covered with epithelium which can grow inwards from its whole circumference.

Bandages themselves tend by their pressure to invert the lids and they are therefore discarded as early as possible.

Occasionally a little knob of granulation tissue springs up on the under surface of the lid and has to be snipped off.

\title{
CROSSED QUADRANT HEMIANOPSIA
}

\author{
BY \\ DR. C. H. FELIX \\ THE HAGUE, HOLLAND
}

OF this eye disease there are only two cases known in literature. I am in a position to add a third.

Mr. V. K., aged 63 years, is suffering from arteriosclerosis and degeneration of the heart. After reading on the evening of April 13, 1924, he retired to bed and awakened the next morning totally blind. The patient was very calm, abnormally calm under these circumstances, whilst his wife was very nervous and wanted to call the doctor immediately. The patient thought that this was not necessary. Noticing that his calmness was very strange under the situation, he answered: "Oh, I have gone through so much that this can also come."

To this very apathetic turn of mind I shall refer later. At about ten o'clock in the morning, when the family doctor visited him, the patient began to see a little again. He could not distinguish the figure of the doctor, but could observe the glistening of his eyeglasses. Gradually the situation became better and after a few days he was able to see large objects and persons as in a mist.

Bronchitis with a disorderly action of the heart kept him in bed for a long time. Not before August 2, 1924, was I able to examine 
him thoroughly. The last two months the sight had much improved, but the family had observed that the sight was something peculiar. "My family don't understand anything about it," he told me on his first visit. "When an illustration is laid before me, I can't tell what it represents, but looking at a small photograph I can recognize it quite well." Reading is impossible, but writing goes better.

The vision of the left eye was $6 / 24$ without glasses, with $+1.25 \mathrm{D}$. sph. $6 / 8$; the vision of the right eye was $6 / 60$ without correction, with $+2 \mathrm{D}$. sph. $6 / 12$ (difficult). The reading of the
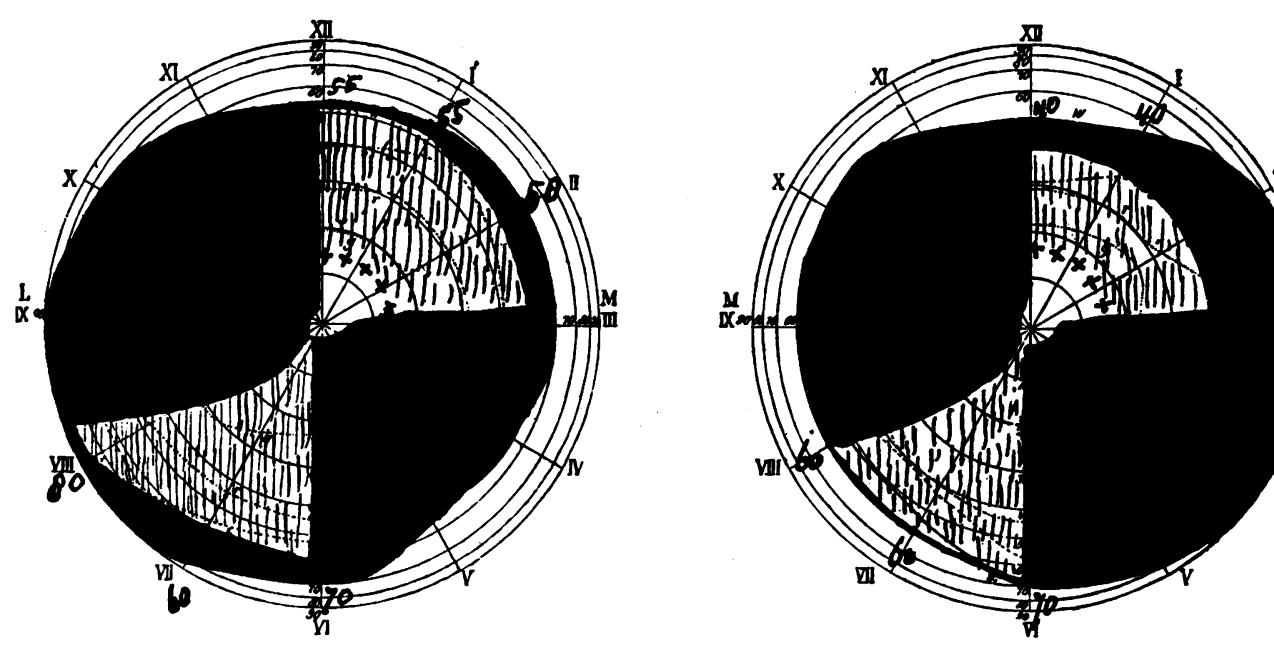

last lines was difficult and only possible by turning the head. In both eyes were found fine opacities of the vitreous. The discs were normal, but there was a slight tortuosity of the retinal vessels. Muscle action was normal, as was also the pupillary reaction.

Examining the visual fields we find a very rare and interesting figure, namely, a crossed quadrant hemianopsia. The left upper scotomata take not only the left upper quadrants but also rather more than one-third part of the next lower quadrants. The right lower scotomata are somewhat larger than the quadrants there. The quadrant scotomata show a sparing of the macula and are absolute. The left lower quadrants of the visual fields are colour blind. In the centre and in the right upper quadrants, which show a peripheral restriction, colours are recognized till about $15^{\circ}$. Outside this there is colour blindness. Twice afterwards the condition of the eyes was examined, but no change was observed.

About the middle of October the patient was subject to apoplexy with hemiplegia of the right side. 
I have only found two cases of this type published: one by Groenouw in 1891 in the Archiv für Psychiatrie, and one by Weymann in the American Journal of Ophthalmology. These cases had complications not observed in my case. It is certainly remarkable that in all three cases the left upper quadrants with the right lower quadrants were blind.

Groenouw's patient had first a left hemiplegia and a left homonymous hemianopsia. Ten months later he was subject to a second apoplectic attack and a crossed quadrant hemianopsia could be observed. The scotomata were not absolute.

Weymann's patient suffered from lues and had violent continual headache. After a sudden collapse with unconsciousness a right hemiplegia appeared and, on examining the visual fields, a crossed quadrant hemianopsia was found. Scotomata were absolute. An energetic antiluetic treatment enabled the patient to resume his work after four months. The scotomata remained unchanged.

In my case the patient had only eye symptoms which make the case more remarkable. I will say a few words about these symptoms, but first I would draw attention to the abnormal calmness which my patient, whose intellect was in perfect order, retained under the terrible ordeal of becoming at once blind.

Such a state of mind at the appearance of cerebral blindness is not unknown in literature, and in the first place was brought to our notice by Redlich and Bonvicini.

These authors have also drawn our attention to another phenomenon of cerebral amaurosis, namely, that some patients are not conscious of their blindness. In some degree resembling this, as Wilbrand and Sänger write in their book, is the state of mind represented in my patient. Förster was the first who was struck by the indolence of his patient and published his observation. Later Groenouw, Probst, Reinhard and Anton have also mentioned this psychical disturbance.

I observed in the middle of October last year a second case of a sudden cerebral blindness in a gentleman, aged 68 years, who also showed this psychical disturbance. This patient, who suffered from a slight dementia senilis, called it a trifle when his wife sitting opposite him at the table and noticing that her husband suddenly looked so strange, with a deadly fright asked him what had happened.

I have put to myself the question: "Was our patient on the morning of April 14 quite amaurotic ?" This cannot be said with certainty, but the possibility of a double homonymous hemianopsia with the sparing of the maculae cannot be excluded. According to Wilbrand and Sänger a sudden double-sided amaurosis, due to intracerebral bleeding, either from embolism or thrombosis of 
the brain vessels should be regarded as an incomplete doublesided hemianopsia. Wilbrand and Sänger have classed the crossed quadrant hemianopsia under the "double-sided homonymous hemianopsia with symmetrical defects of the visual fields."

The symptoms of this disease can only be explained by a sudden affection of both hemispheres of the brain. In what part of the visual paths must we seek the disease? Of course behind the chiasma; therefore in the optic tract, the primary optic ganglia, the optic radiation, or cortical centre of vision.

The sparing of the macula on both sides in my case militates against the presence of the lesions in the optic tracts.

Wernicke's sign, the hemianopic reaction of the pupil, cannot throw any light; more help may be obtained from the absence of a partial atrophy of both discs.

Dufour has pointed out that the tract scotomata are seen black by the patient, while in cortical and subcortical lesions the patient is no more conscious of his visual field defect than a healthy person of his blind spot. My patient seemed to be unconscious of his scotomata.

Cortical and subcortical hemianopsia come on more suddenly, tract hemianopsia most often slowly as a result of the pressure of a tumour.

A double-sided lesion of the primary optic ganglia, near the posterior part of the internal capsule, should, I suppose, nearly always be accompanied by other symptoms in consequence of a disease of the important neighbouring parts of the brain. Also since only the optic paths are affected, I feel inclined to conclude that most likely the lesions are to be found in the cortical centres of vision or down in Gratiolet's optic radiation, and caused by a disease in one of the branches of the calcarine arteries.

I think we can determine the place more exactly if we accept the opinion of Henschen about the projection of the different parts of the visual field upon the centre of the vision. Following Henschen, the lowest visual field quadrant is localized in the upper lip of the calcarine fissure, and the upper quadrant in the lower lip.

So the foci are to be found: (1) in the left hemisphere in the upper lip of the calcarine fissure; and (2) in the right hemisphere in the lower lip of the calcarine fissure. For the right quadrant scotomata are caused by a lesion in the left hemisphere and while these scotomata are situated in the lowest part of the visual field, the lesion is to be found in the upper calcarine lip of the left centre of vision; the left quadrant scotomata are caused by a lesion in the right hemisphere, and, while these scotomata are situated in the upper part of the visual field, the lesion is to be found in the lower calcarine lip of the right centre of vision. 
Finally, we will consider the question whether the double lesion is the result of a haemorrhage, embolism or thrombosis. Of course we cannot give here a positive answer. In Wilbrand and Sänger's handbook we find some differential diagnostic signs.

(a) The unconciousness in apoplexy is the deepest, in embolism it is present but not so deep and lasts a short time, while in thrombosis it is often absent or very superficial.

(b) The course of the three diseases is different. Thrombosis runs the longest course in consequence of the disease of the brain vessels, i.e., headache, dizziness, decrease of memory and intellect. Apoplexy is often preceded by haemorrhages of the retina and by dizziness. Embolism occurs most suddenly and without preceding brain symptoms.

My patient never presented signs of an approaching braindisease. It is possible that the nervousness, weariness and dullness, my patient complained of, may have had something to do with arteriosclerosis of the brain vessels. I cannot decide if there had been unconsciousness on the night of April 14. The apoplexy later on indicates disease of the brain vessels. A little haemorrhage may occur without unconsciousness, but two bleedings appearing at the same moment in the region of both calcarine arteries would be a very rare casualty.

Wilbrand and Sänger have published a case of sudden doublesided blindness from simultaneous apoplexy in both occipital lobes of the brain. The report of the autopsy, however, makes mention of many little haemorrhages not only in the occipital lobes but also in other parts of the brain.

A simpler explanation may be given here: A small thrombus, not closing the vessel, is formed just on the place where both deep cerebral arteries arise from the basilar artery. This thrombus has then been divided in two parts which are carried by the blood stream to the calcarine arteries.

Summary : we have here a case of crossed quadrant hemianopsia starting from a double-sided homonymous hemianopsia with probable sparing of the maculae in a patient who showed no other cerebral symptom. This makes my case more remarkable than the two other cases. It seems to be unique. 